\title{
WEBTOP: WEB-BASED INTERACTIVE 3D OPTICS AND WAVES SIMULATIONS
}

\author{
Taha Mzoughi, John T Foley, S. Davis Herring, Matt Morris, Ben Wyser
}

\begin{abstract}
WebTOP is a three-dimensional, interactive computer graphics system developed at Mississippi State University to help students learn about optics and waves. It has been used to help teach undergraduate introductory physics and upper-level optics classes. Currently, it comprises sixteen modules spanning eight different subject areas. The subject areas are waves, geometrical optics, reflection and refraction, polarization, interference, diffraction, lasers, and scattering. WebTOP simulations have the following characteristics. First, they are three dimensional, i.e., they have navigation controls that allow the user to rotate the scene, pan it, or zoom into it, in order to view it from any desired orientation. Secondly, they are interactive. The user can change the parameters either by typing in the desired values into the appropriate text entry box, or by using his/her mouse cursor to move the appropriate widget in the scene. Thirdly, the simulations are animated, for those phenomena for which animation is appropriate. Furthermore, the simulations include VCR-type controls that allow the user to record his/her interaction with the simulation for later retrieval, viewing, and editing. Finally, these modules run inside a web browser. They can be run from our website, http://webtop.msstate.edu, or they can be downloaded from this website and run on the user's local machine. This paper provides an overview of WebTOP and a description of each of the modules.
\end{abstract}

\section{KEYWORDS}

Optics, waves, scripts, simulations, VRML, Java, N-slit, single, slit, reflection, refraction, lenses, stops, eye, polarization, Michelson, Interferometer, Fabry-Perot, Etalon, Fraunhofer, Rayleigh, resolution, Fresnel, circular, aperture, transmission, grating, scattering, lasers

\section{INTRODUCTION}

The Optics Project on the Web (WebTOP) is a Web-based 3D interactive computer graphics system that simulates optical and wave phenomena (Foley, 2000, Vidimce 2000). Its purpose is to help teachers teach and students learn about waves and optics. The current version includes sixteen modules: Waves, Lenses, The Eye, Reflection and Refraction/Vectorial, Reflection and Refraction/Waves Two Media, Reflection and Refraction/Waves Three Media, Polarization, Michelson Interferometer, Fabry-Perot Etalon, Fraunhofer N-Slit, Transmission Grating, Rayleigh Resolution, Fresnel Single Slit, Fresnel Circular Aperture, Scattering, and Lasers. Each of the modules includes an interactive simulation, an overview of the relevant theory, a showcase of examples, and a list of suggested exercises.

The WebTOP simulations are implemented using the Virtual Reality Modeling Language (VRML) (Rikk, 1997), Java and the External Authoring Interface (Phelps, 2003). They run in Microsoft's Internet Explorer Web browser with the Blaxxun Contact 5.0 VRML browser plug-in installed (Blaxxun, 2003). WebTOP simulations include VCR-type controls that allow users to record their interaction with the simulation in the form of small XML scripts (XML, 2003). The scripts are humanreadable and easily edited. They can be used for viewing a pre-recorded session, setting up example web pages, or for simply initializing modules.

WebTOP is used to help teach both upper-level undergraduate (junior/senior) optics courses and/or the wave and optics parts of introductory physics courses (both calculus-based and algebra-based) in more 
than a dozen universities. In this paper we first describe the features of a typical module, and then discuss the capabilities of each module. Finally, the use of WebTOP in classroom settings is described.

\section{FEATURES OF A WEBTOP MODULE}

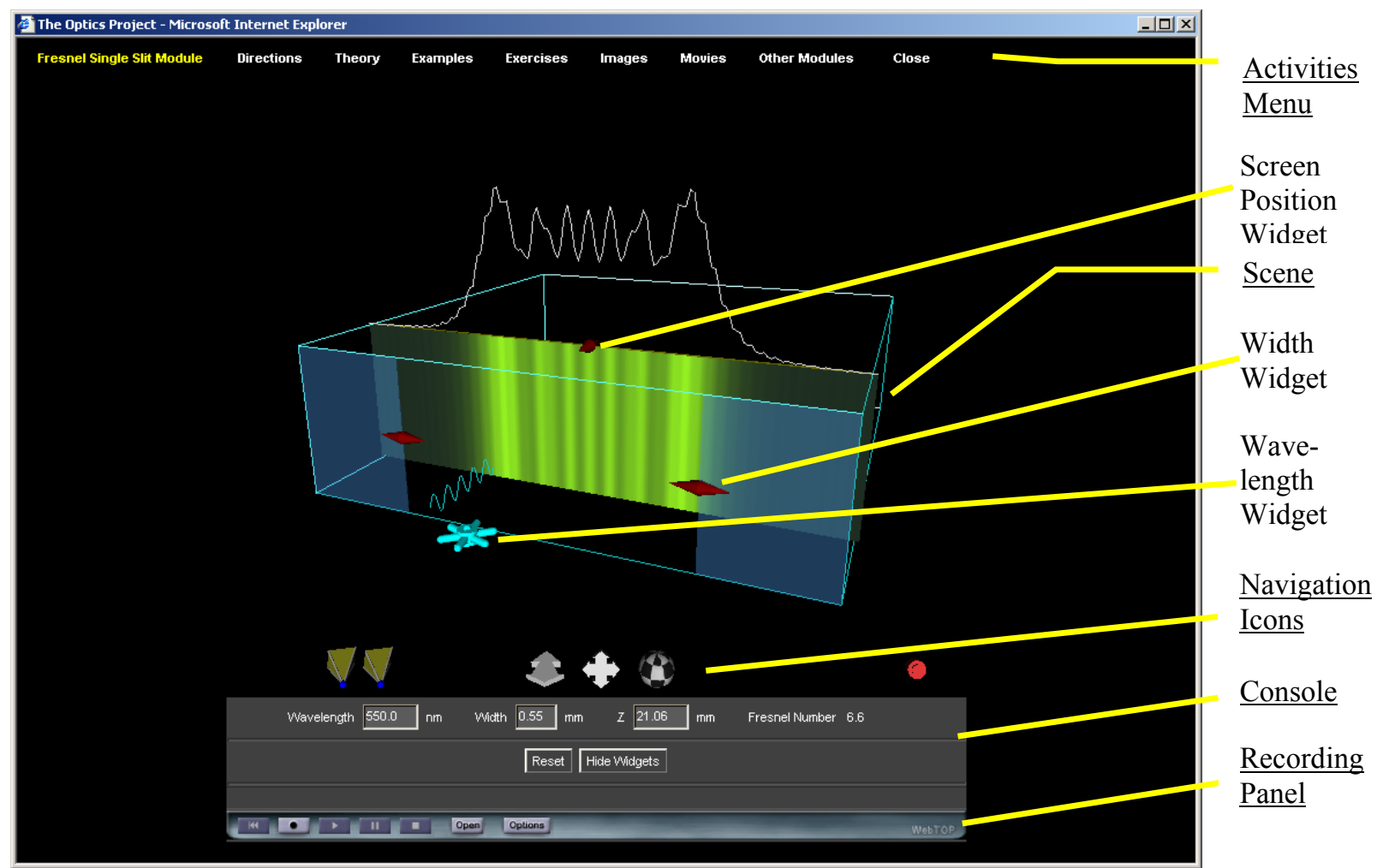

Figure 1. The WebTOP window for the Fresnel Single Slit Module. Underlined labels indicate the five basic parts of the window; the other labels point to the widgets used in the module

A typical WebTOP module window is shown in Figure 1. It has five basic parts: the Scene, the Navigation Icons, the Console, the Recording Panel, and the Activities Menu. Each of these will be described in the following sections.

\section{The Scene}

The Scene is the interactive 3D simulation itself and occupies the largest part of the Web page. It usually consists of a light source, a variety of optical elements, and an observation screen. The parameters of these items can be modified either by direct manipulation of the "widgets" in the scene, or by typing the desired parameter values into the appropriate text entry boxes on the Console.

For example, in the Fresnel Single Slit module shown in Figure 1, the scene consists of a single slit, an observation screen, and three kinds of widgets that allow the user to change the parameters of the simulation. In this module a monochromatic plane wave of wavelength $\lambda$ is normally incident upon a slit of width $w$. The resulting diffraction pattern is observed on an observation screen that is a distance $z$ from the plane that contains the slit. Spinning the Wavelength widget changes the wavelength. Pulling on the Width widget changes the width of the aperture. Pulling on the Screen widget changes $z$.

\section{The Navigation Icons}

The five navigation icons allow the user to control the appearance of the scene. They are shown in Figure 2. The Viewpoint icon (the Fresnel Single Slit module, shown in Figure 1 has two) returns the scene to predefined positions. The Zoom icon allows the user to zoom into or out of the scene. The Pan 
icon allows the user to translate the scene left /right or up/down. The Rotate icon allows the user to rotate the scene. The Hide Icons icon allows the user to hide and show all the other navigation icons.

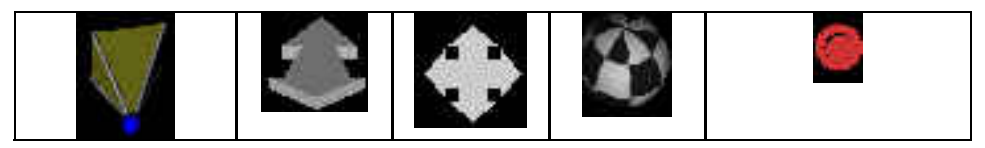

Figure 2. The Navigation Icons, from left to right: Viewpoint, Zoom, Pan, Rotate, Hide Icons

\section{The Console}

The Console is near the bottom of the window (see Figure 1). It has three functions. First, it tells the user the current values of the input parameters, and other important quantities. For example, the Fresnel Single Slit module, the Console is reporting that the values of the input parameters are wavelength $=$ $550 \mathrm{~nm}$, slit width $=0.60 \mathrm{~mm}$, and $z=25 \mathrm{~mm}$, and that the value of the Fresnel number is 6.6. The second purpose of the console is that it allows users to change the input parameters. The third thing the Console does is that it provides context sensitive help messages on the use of the widgets, and read-outs from sensors in the scene. For example, if the mouse cursor is placed over the observation screen in Fig. 1 , the console reports the corresponding position on the screen and intensity at that point.

\section{The Recording Panel}

The Recording Panel is at the bottom of the window. It contains VCR-like controls that allow the user to record a WebTOP session, store it in the form of a script, and then play the script back at a later time. Scripts can also be used to provide example web pages or to set up the initial parameters for a module. The names of the buttons in the Recording panel are, from left to right: Reset, Record, Play, Pause, Stop, and Open.

There are two modes of operation of the recording feature of WebTOP, the RAM-Mode and the Disk Mode. In the RAM mode, the user can record a session and replay it but cannot save it to disk. The recording is lost when the user closes the browser. The disk mode allows the user to additionally save the sessions to the computer hard disk, and to open and play sessions that are on disk. In order to use the Disk mode you must install the WebTOP recording library on your computer. When placed on a website, WebTOP scripts can be replayed even by users who choose not to install the library.

\section{The Activities Menu}

The Activities Menu is at the top of the window. It lists the five activities available in WebTOP: the title of the module (Fresnel Single Slit in this case), Directions, Theory, Examples, and Exercises. It also provides a link to the other modules. The title provides access to the interactive simulation itself. The Directions activity contains documentation on how to interact with that particular module. The Theory section presents an overview of the physics being simulated in the module. The Examples section is a Web page that contains descriptions of and links to previously recorded WebTOP sessions in the form of scripts. The scripts are loaded by clicking on the name of the example, and then played by clicking on the Play button on the Recording Panel. The Exercises section provides exercises for the user to try. These are inquiry-based exercises in that the user is asked to interact with the module, observe how the simulation changes, and then come up with an explanation for what is happening. Clicking on Other Modules displays a list of the other modules that the user can access.

\section{WEBTOP MODULES}

In the following we will provide a short description of each of the WebTOP modules.

\section{The Waves Module}

The Waves module simulates waves in a ripple tank. The user can place one or more monochromatic point sources and/or monochromatic line sources on the water surface. The module shows the resultant 
disturbance, either as a still picture or as traveling waves. For each point source the user can interactively vary the amplitude, wavelength, and initial phase of the wave it generates, as well as the position of the source. For each line source the user the user can vary the amplitude, wavelength, direction of propagation, and initial phase of the wave it generates. Figure 3 shows the interference pattern due to two point sources.

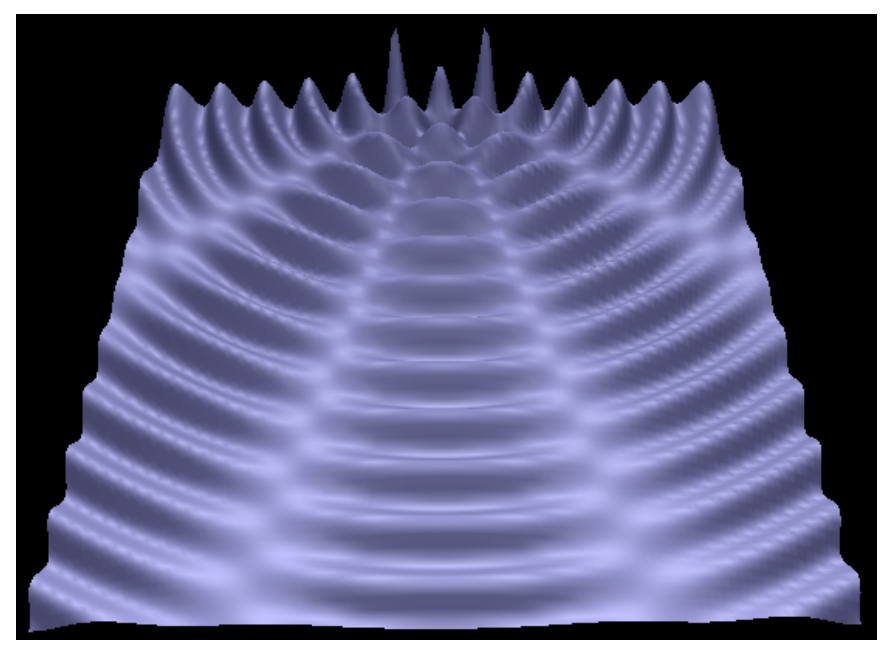

Figure 3. The Waves module. Interference pattern due to two point sources

\section{The Lenses Module}

Figure 4 illustrates the Lenses module. This module simulates the behavior of light as it passes through lenses and stops on an optical bench. The user can use either a two-dimensional object (five point sources in the shape of a T) or a three-dimensional object (nine point sources on the a plane that can be rotated). The user can put several lenses and stops on the bench; the position, diameter and focal length of each lens can be varied interactively, as can the position and diameter of each stop. Each point source on the object emits a large number of rays at random angles, and these rays travel through the system according to the laws of geometrical optics. A movable observation screen allows the user to see the ray distribution in any plane perpendicular to the axis of the bench.

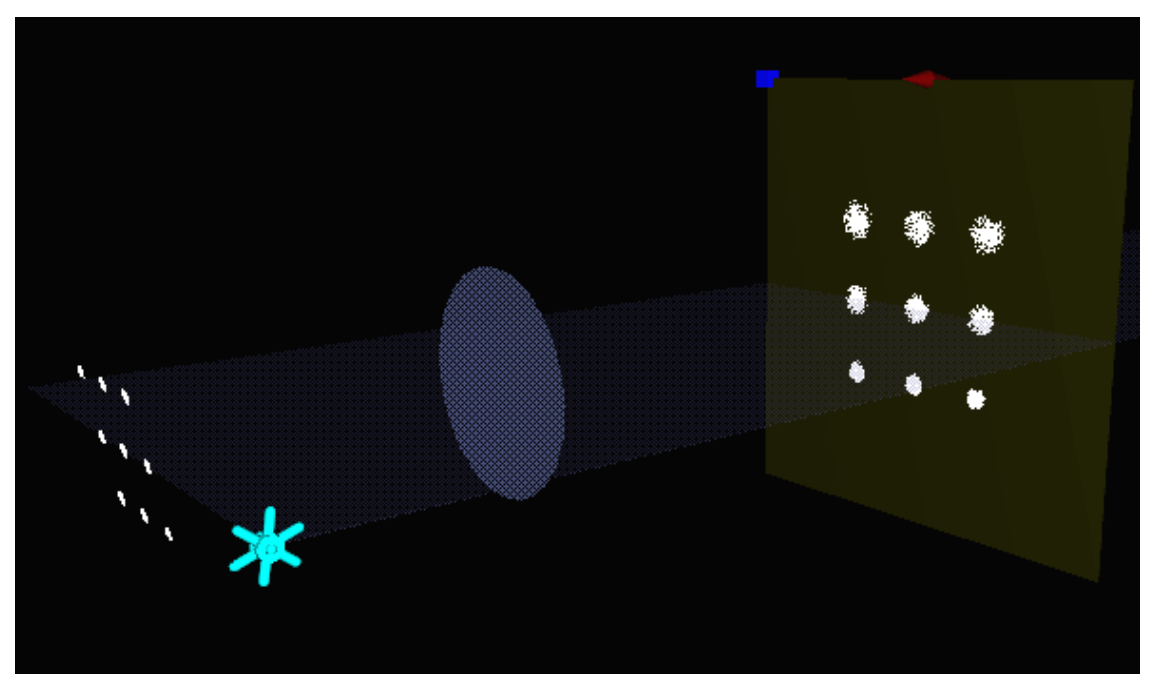

Figure 4. The Lenses module. A three dimensional object is being projected on a screen by a converging lens 


\section{The Eye Module}

This module simulates image formation by the human eye with either normal vision or vision that needs corrective lenses. The user controls the position of the object being observed, the length of the eye, and the minimum and maximum focal lengths of the eye. The eye automatically accommodates as the object is moved. In addition, the module allows the user to place a corrective lens (either an eyeglass lens or a contact lens) in front of the eye to see the effect that this has on the image formed. Since the object distance and the eye length scales are typically quite different, two views are depicted simultaneously: a far away view which shows the object and a small eye, and a close-up view that shows the image on the retina, the corrective lens (if any) and the rays of light entering the corrective lens-eye combination.

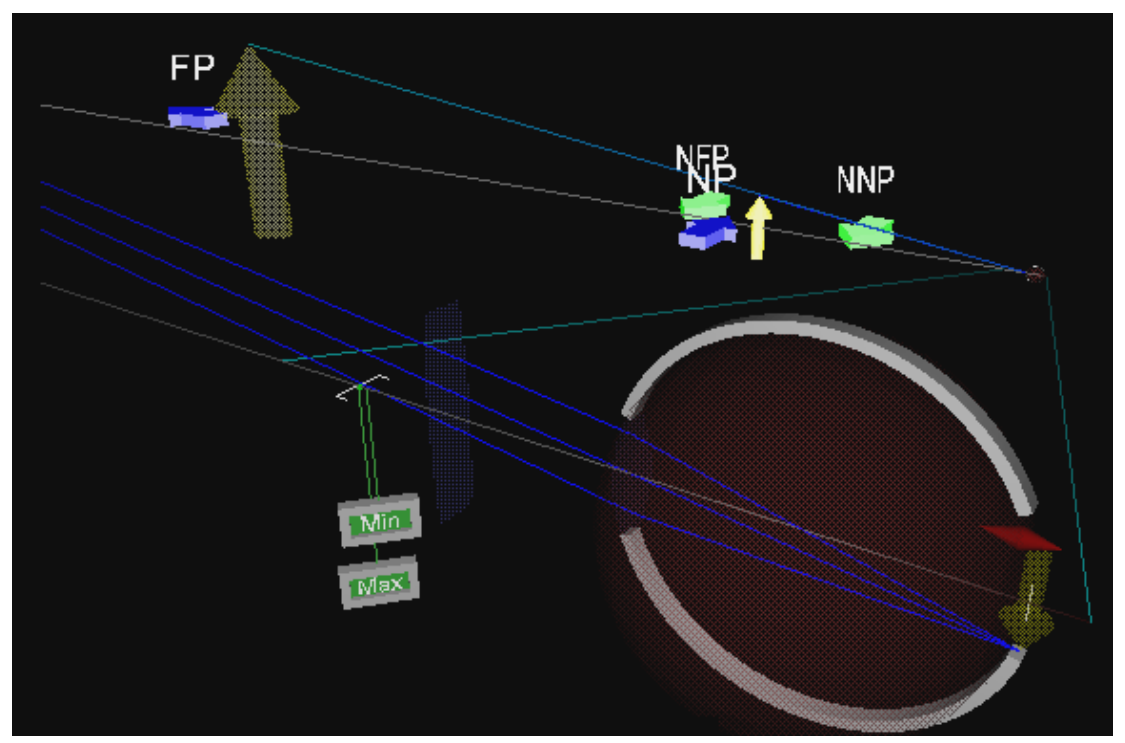

Figure 5. The Eye module

\section{The Reflection and Refraction/Vectorial Module}

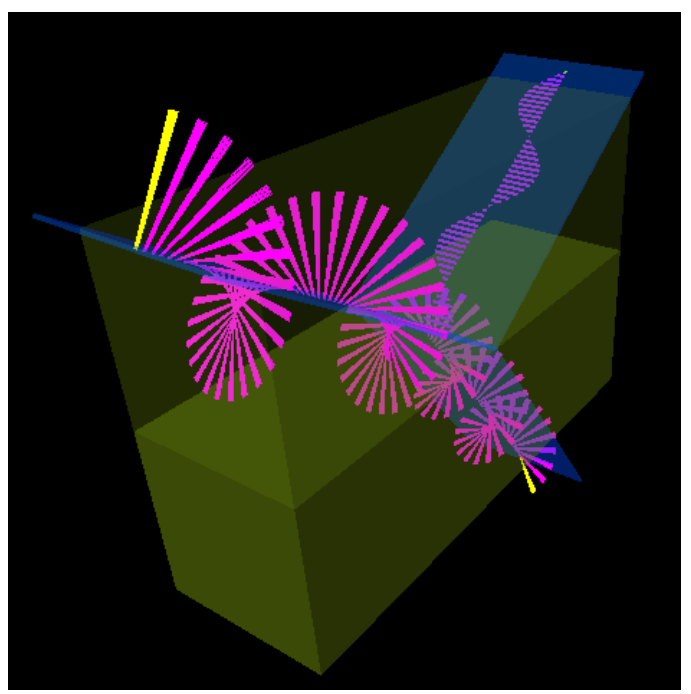

Figure 6. The Reflection and Refraction/Vectorial module. Circularly polarized light is incident from air onto glass at Brewster's angle

In this module a monochromatic plane wave is incident upon a planar interface that separates two homogeneous media. The user can choose the incident electric field to be either completely polarized or to be unpolarized. In the completely polarized case, the user can interactively vary the wavelength, 
angle of incidence of the incident wave, the amplitudes of the two components and the phase difference between them. The corresponding time-varying incident, reflected, and transmitted (refracted) electric field vectors along the corresponding ray paths are displayed on the screen. The user can select which components of the electric field are displayed.

\section{The Reflection and Refraction/Waves Two Media Module}

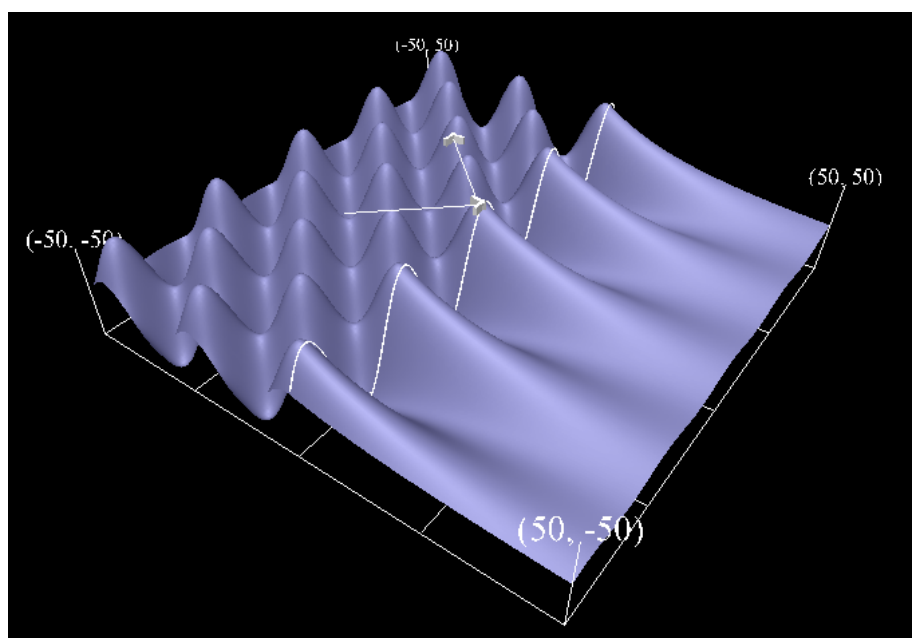

Figure 7. The Reflection and Refraction/Waves Two Media module. S-polarized light is incident from glass (on the left) onto air at an angle of incidence greater than the critical angle

This module simulates a monochromatic plane wave of s-polarized light incident upon a planar interface that separates two homogeneous media. Instead of depicting the electric field vectors at a fixed set of points along one set of incident, reflected and transmitted rays, as is done in the Reflection and Refraction/Vectorial module, the wave functions for the electric fields on both sides of the interface are depicted as waves in a ripple tank. On one side of the "borderline" between the two media, the user can display either the incident wave, or the reflected wave, or the superposition of the two. On the side of the borderline, the transmitted wave is displayed. The user controls the wavelength, amplitude, and angle of incidence of the incident wave, and the indices of refraction of the two media.

\section{The Reflection and Refraction/Waves Three-Media Module}

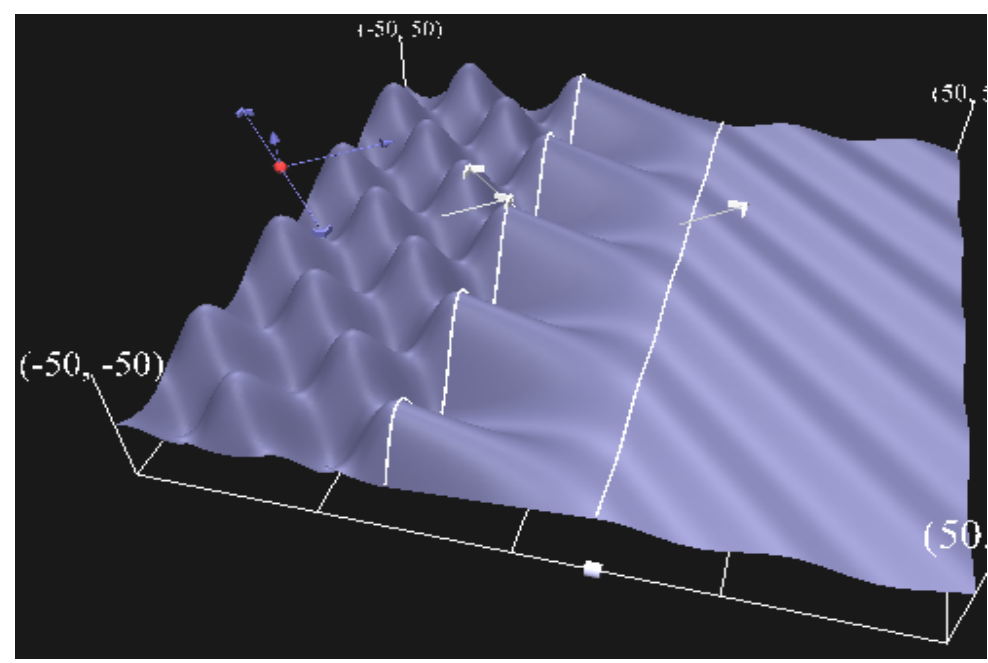

Figure 8. The Reflection and Refraction/Waves Three Media module. Frustrated total internal reflection is depicted 
This module is an extension of the Waves Two Media module. It simulates a monochromatic plane wave of s-polarized light as it travels through three different homogeneous media. The wave functions for the electric fields in each of the media are depicted as waves in a ripple tank. The user controls the wavelength, amplitude, and angle of incidence of the incident wave, the indices of refraction of each of the three media, and the thickness of the second medium.

\section{The Polarization Module}

The Polarization module simulates the propagation of the electric field vectors of either a completely polarized or unpolarized monochromatic plane wave of light and the effects of various optical elements (linear polarizers and wave plates) on the corresponding electric field vectors. The user controls the properties of the incident field, and the type, location, and characteristics of the optical elements being used. For a linear polarizer the user can vary its position, and the angle that its transmission axis (TA) makes with the positive $\mathrm{x}$-axis. For a wave plate the user can vary its position, the angle that its fast axis makes with the positive $\mathrm{x}$-axis, and its thickness in units of the incident wavelength.

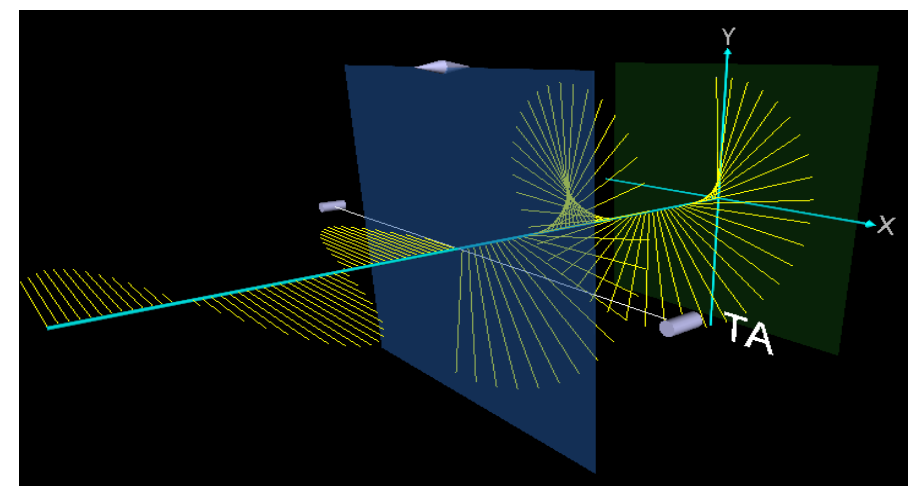

Figure 9. The Polarization module

\section{The Michelson Interferometer Module}

In this module light from a monochromatic point source is incident upon a Michelson interferometer. The resultant intensity pattern is displayed on an observation screen, and a graph of the intensity as a function of position across the center of the pattern is displayed above the observation screen. The user can vary the wavelength of the incident light, the rotation angle of the tilt mirror, and position of the translation mirror.

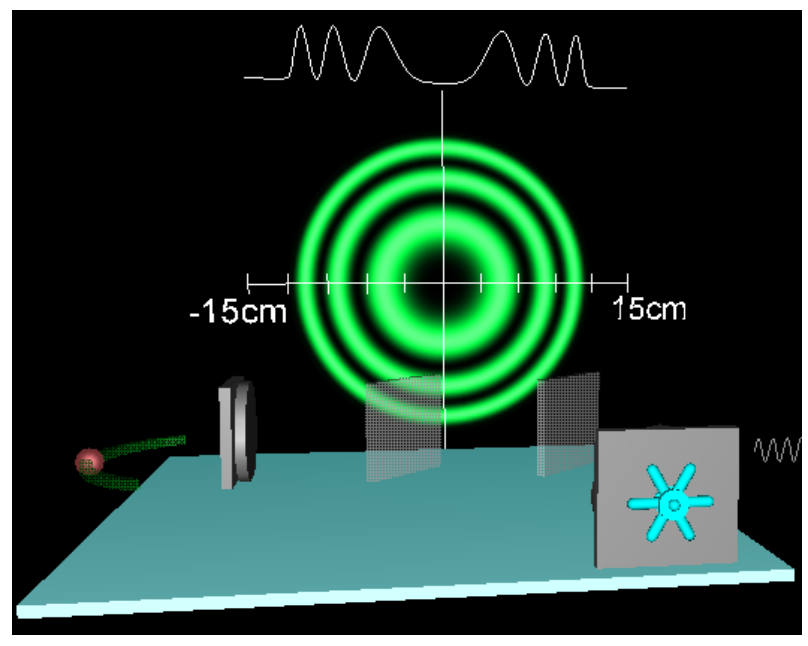

Figure 10. Michelson Interferometer module

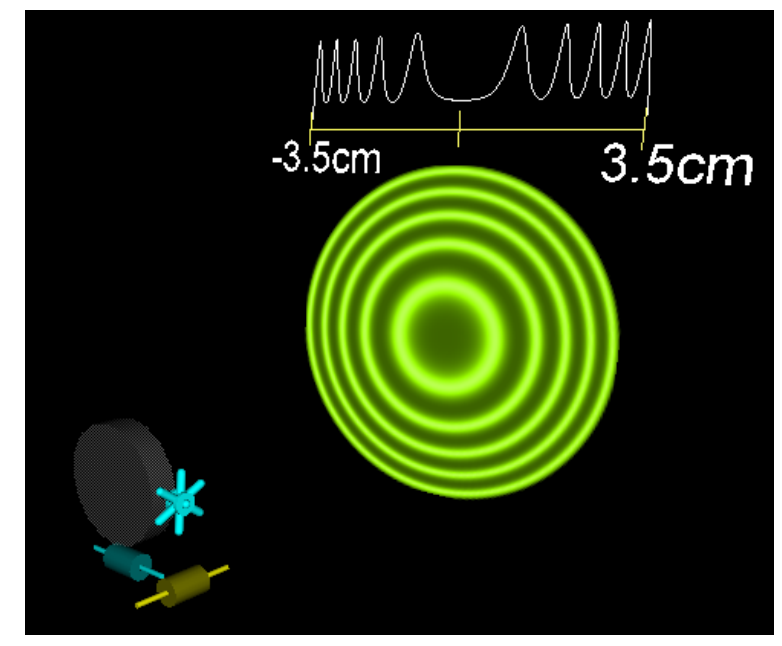

Figure 11. Fabry-Perot Etalon module 


\section{The Fabry-Perot Etalon Module}

In this module light from a monochromatic point source is incident upon a dielectric slab whose surfaces are coated with a reflective coating. The resultant interference pattern is displayed on an observation screen, and a graph of the intensity as a function of position across the across the center of the pattern is displayed above the observation screen. The user can vary the wavelength of the incident light, the reflectivity of the faces of the slab, the thickness of the slab, and the index of refraction of the slab.

\section{The Fraunhofer N-Slit Module}

The Fraunhofer N-Slit module simulates a plane wave of monochromatic light of wavelength $\lambda$ that is normally incident upon a plane that contains $N$ identical slits $(N \geq 1)$. The resulting intensity pattern is displayed on an observation screen, and a graph of the intensity as a function of position across the screen is displayed above the observation screen. The user can vary the wavelength of the incident light, the number of slits, the slit width, the distance between consecutive slits, and the distance from the slit plane to the observation plane. Moving the cursor over the screen causes the intensity at that point to be displayed on the console.

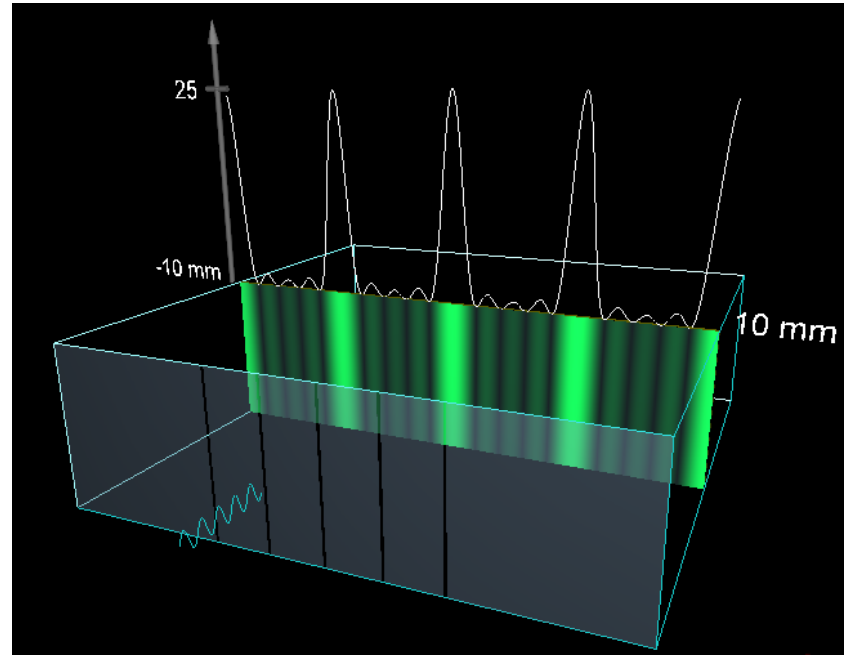

Figure 12. Fraunhofer N-Slit module

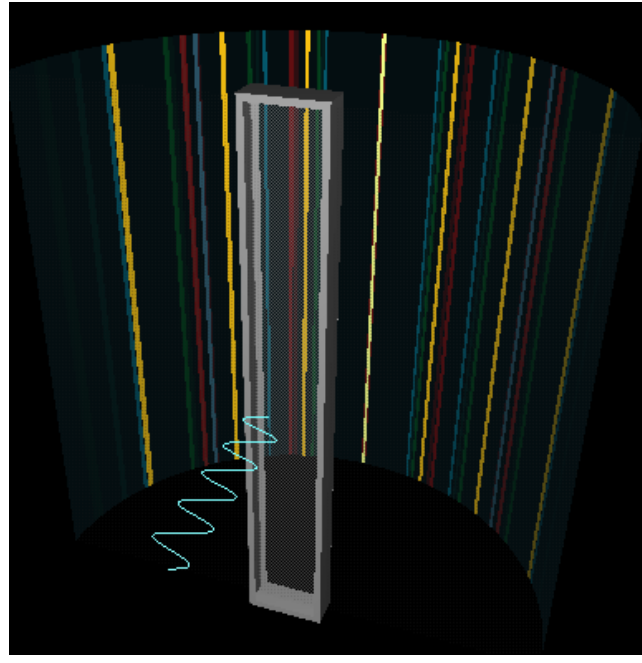

Figure 13. Transmission Grating module

\section{The Transmission Grating Module}

In this module, a polychromatic plane wave is incident upon a transmission diffraction grating, and the resulting intensity pattern is displayed on a semicircular observation screen. The user can select the spectrum of the incident light to be that of a predefined standard gas discharge tube (hydrogen, helium, sodium, or mercury), or can create a spectrum by entering various wavelengths. The mouse cursor acts as a sensor and, when placed over the observation screen, allows the user to read the angular position of the cursor (and hence the position of the various diffracted orders on the screen). 


\section{The Rayleigh Resolution Module}

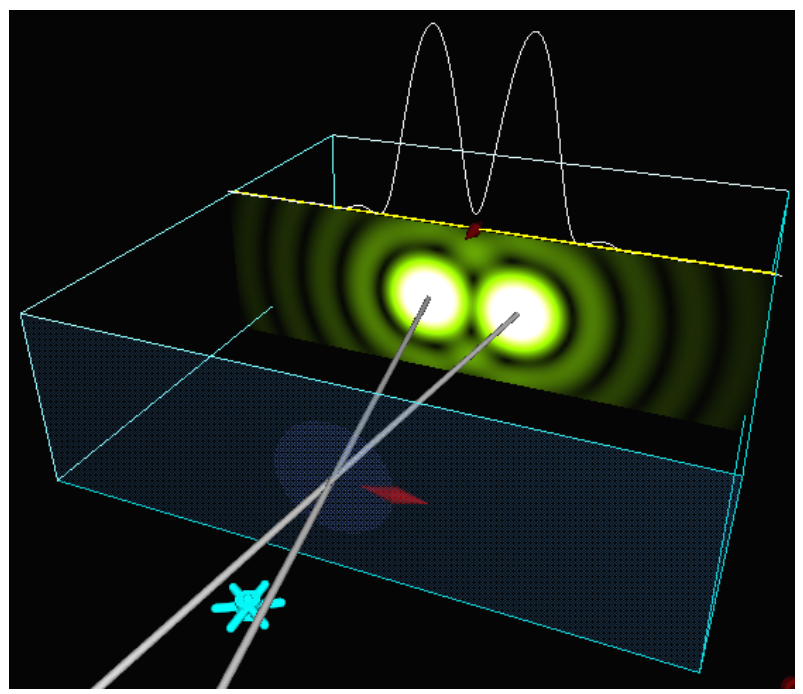

Figure 14. The Rayleigh Resolution module

In this module monochromatic light from two distant point sources separated by a small angle is incident upon a lens. The resulting intensity pattern is viewed on an observation screen positioned in the focal plane of the lens, and a graph of the intensity as a function of position across the center of the pattern is displayed above the observation screen. The user can vary the wavelength $\lambda$ of the light, the angle $\theta$ between the sources, and the diameter $D$ of the lens. The Console displays, in addition to the values of $\lambda, D$, and $\theta$, the value of the minimum angle for which (according to Rayleigh) the two images can be resolved.

\section{The Fresnel Single Slit Module}

In this module a monochromatic plane wave light is normally incident upon a single slit. The resulting diffraction pattern is observed on an observation screen, and a graph of the intensity as a function of position across the screen is displayed above the observation screen. The user can vary the wavelength of the light, the width of the slit, and the distance from the slit to the observation screen. The parameter ranges are such that the user can explore the Fresnel region, and the beginning of the Fraunhofer region. Moving the cursor over the screen causes the intensity at that point to be displayed on the console.

\section{The Fresnel Circular Aperture Module}

In this module a monochromatic plane wave light is normally incident upon a circular aperture. The resulting diffraction pattern is observed on an observation screen, and a graph of the intensity as a function of position across the screen is displayed above the observation screen. The user can vary the wavelength of the light, the diameter of the aperture, and the distance from the aperture to the observation screen. Moving the cursor over the screen causes the intensity at that point to be displayed on the console. The parameter ranges are such that the user can explore the Fresnel region, and the beginning of the Fraunhofer region. 


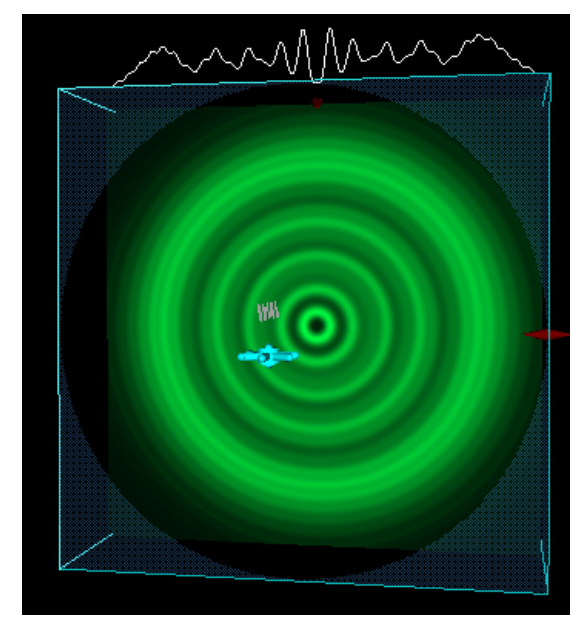

Figure 15. The Fresnel Circular Aperture module

\section{The Scattering Module}

This module simulates the non-resonant scattering of light from an atom. The atom is located at the origin, and the incident light is traveling along the negative y-axis in the positive y-direction. An oscillating vector depicts the induced dipole moment of the atom. Scattered electric field vectors are depicted at sets of observation points along three different axes: the positive x-axis, the positive $\mathrm{z}$-axis, and movable axis whose angular position can be varied by the user. The user can choose the incident electric field to be either linearly polarized or unpolarized. When the incident light is linearly polarized, the user can change the amplitudes of the two components of the electric field vector, and the wavelength of the light.

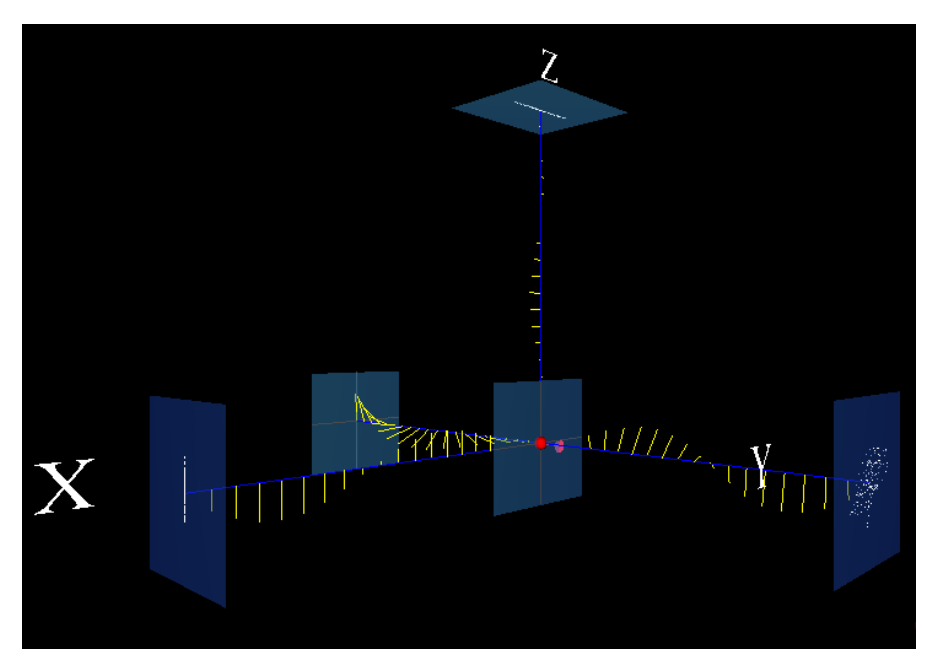

Figure 16. The Scattering Module

\section{The Lasers Module}

This module simulates the behavior of an optical resonator oscillating in one of the following four transverse modes (the user selects which one): $\mathrm{TEM}_{00}, \mathrm{TEM}_{10}, \mathrm{TEM}_{01}$, or TEM $\mathrm{TE}_{11}$. The scene consists of a cavity that has a spherical mirror on each end. The resulting intensity pattern is displayed on an observation screen, and a graph of the intensity as a function of position across the center of the pattern is displayed above the observation screen. The user can vary the wavelength of the light, the radius of curvature of each of the mirrors, the length of the cavity, and the position of the observation screen. 


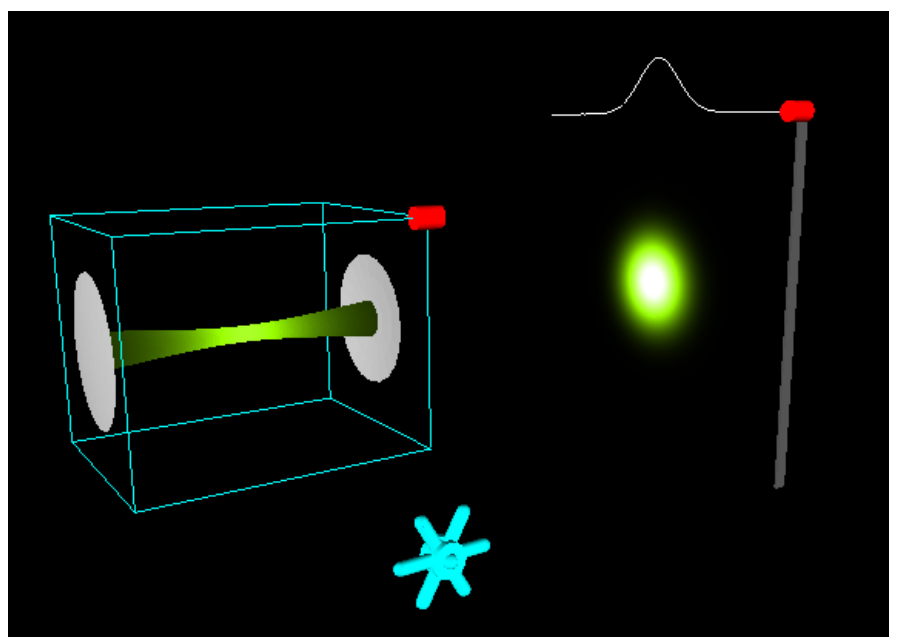

Figure 17. The Lasers module

\section{USING WEBTOP FOR TEACHING AND LEARNING}

WebTOP is designed to be flexible, allowing faculty and students to use it in a variety of ways. In the following, we will describe some of the uses we can think of, as well as results from student surveys stemming from our use of WebTOP in introductory physics courses.

\section{Classroom Use}

WebTOP can be used for in-class demonstrations. Its three-dimensional nature allows the presenter to show the phenomenon from the most advantageous viewpoint. The ability to change the values of all the relevant parameters in a simulation allows the instructor to show, in real time, the effect of changing each parameter in a way that can be seen clearly by the students, even in large lecture halls. More importantly, using WebTOP to present material lends itself to "What will happen if ..." kinds of questions, providing an ideal mechanism for creating an active learning environment (Sokoloff, 1997).

Furthermore, WebTOP can be used for homework. Students can be asked to complete homework sets in which they are required to use WebTOP. They can be asked to analyze certain situations, produce numerical or qualitative answers to questions, and then use WebTOP to simulate the situation and check their answers. The students can be asked to turn in their written answers to the questions, relevant screen captures from WebTOP modules, or WebTOP scripts.

WebTOP can also be used to supplement laboratory activities. It can be used in pre-lab activities to help explain the phenomenon that is going to be investigated in the lab, or it can be used during the lab to compare actual data to the simulation results and to help draw inferences when equipment limitations occur.

In addition, WebTOP can be used to develop online tutorials. With scripts, the user can use a WebTOP module to illustrate numerous phenomena by simply setting up the initial parameters for the module, or by recording a set of actions.

Finally, WebTOP can be used for student projects. Students can work in teams or individually on particular problems and use WebTOP to help illustrate their presentations and reports.

\section{Content Considerations}

While all WebTOP modules are useful for upper level undergraduate optics courses only about half are applicable to introductory courses. Some possible uses for introductory classes include the Waves module to show the class circular waves propagating outwards from a point source, and to show the interference of waves from two point sources. The Reflection and Refraction/Two Media module can be 
used to show the reflection and refraction of waves at a planar interface. The Lenses module can be used to show the students how single converging and diverging lenses bend light rays to form images, and how combinations of lenses work together to form images. The Eye module can be used to reinforce the concepts of the far point and near point of an eye, and to simulate putting eyeglasses or contact lenses on a nearsighted or farsighted eye. The Polarization module can be used to depict the electric field vectors of linearly polarized light or unpolarized light, and to simulate the effect of a system of linear polarizers on such fields. The $\mathrm{N}$-slit module can be used to simulate single slit diffraction, the diffraction from two or more slits, and the effects of changing slit widths, slit separations, and the number of slits. The Rayleigh Resolution module shows how decreasing the angle between two point sources affects the ability of a lens to resolve them, and how changing the lens diameter affects resolution. The Scattering module can be used to show polarization by scattering, and the wavelength dependence of Rayleigh scattering.

\section{Student Evaluations}

At Mississippi State University, WebTOP was used in two types of introductory physics classes: calculus-based and algebra-based. The calculus-based class comprised engineering, physics, science and math majors. The book used was "Physics for Scientists and Engineers", 5th edition, by Raymond A. Serway and Robert Beichner (Serway, 2000). The algebra-based course comprised mainly architecture, biology, and pre-med majors. The book used was "Physics: Principles with Applications", Fifth Edition, by Douglas Giancoli (Giancoli, 1998).

WebTOP was used in these classes for both in-class demonstrations and homework. Results from anonymous surveys collected at the end of the semester are provided in Tables 1 and 2.

Table 1. Student responses to "How useful did you find the WebTOP demonstrations during class for visualizing and understanding the optical phenomena in this course?" (Scale: $5=$ very useful, $1=$ not at all useful.)

\begin{tabular}{|l|r|r|r|}
\hline Class & Mean & Standard. Deviation & Number of Students \\
\hline Calculus-Based, Spring 2001, Sec. 1 & 4.56 & 0.69 & 43 \\
\hline Calculus-Based, Spring 2001, Sec. 2 & 4.53 & 0.57 & 55 \\
\hline Calculus-Based, Fall 2001 Sec. 1 & 4.51 & 0.54 & 47 \\
\hline Algebra-Based, Spring 2002 Sec. 1 & 4.54 & 0.63 & 52 \\
\hline Algebra-Based, Spring 2002 Sec. 2 & 4.51 & 0.64 & 53 \\
\hline
\end{tabular}

Table 2. Student responses to "How useful did you find the WebTOP homework during class for visualizing and understanding the optical phenomena in this course?" (Scale: $5=$ very useful, $1=$ not at all useful.)

\begin{tabular}{|l|r|r|r|}
\hline Class & Mean & Standard. Deviation & Number of Students \\
\hline Calculus-Based, Spring 2001, Sec. 1 & 4.37 & 0.72 & 43 \\
\hline Calculus-Based, Spring 2001, Sec. 2 & 4.27 & 0.72 & 55 \\
\hline Calculus-Based, Fall 2001 Sec. 1 & 4.17 & 0.91 & 47 \\
\hline Algebra-Based, Spring 2002 Sec. 1 & 4.17 & 0.99 & 52 \\
\hline Algebra-Based, Spring 2002 Sec. 2 & 4.33 & 0.73 & 53 \\
\hline
\end{tabular}

The results clearly show that students found WebTOP is very useful for both classroom demonstrations and homework. 


\section{CONCLUSION}

In teaching and learning optics, we often deal with content that is difficult to understand without good visualizations. WebTOP provides a means to easily visualize phenomena in $3 \mathrm{D}$ allowing users to both look at phenomena from different angles and answer "what if" type questions. WebTOP has been shown to be an effective tool for classroom demonstrations, homework, and for developing online tutorials. It can also be used to help supplement laboratory activities and for student projects. Yet, WebTOP is not a finished product. We have plans to develop more curriculum material based on WebTOP modules and to develop more modules. We also plan to make WebTOP open source. This will enable users to modify and expand WebTOP, and to adapt its techniques to their particular projects. For more details about WebTOP's progress visit the website at: http://webtop.msstate.edu

\section{REFERENCES}

Blaxxun (2003), the Blaxxun Contact 5.0 VRML browser plug-in can be obtained from the Blaxxun Web site; http://www.blaxxun.com/ accessed April 11, 2003.

Foley, J. T. (2000), Vidimce, K., Banks, D. C., and Mzoughi, T., "WebTOP: Interactive 3D Optics Simulations on the Web," in Education and Training in Optics and Photonics, ed. by J. SanchezMondragon, SPIE 3831, 214-222.

Giancoli, D.C. (1998), “Physics”, 5th. Ed., Prentice-Hall Publishing, New Jersey.

Phelps, A. (2003), Mason, E., External Authoring Interface Working Group. Retrieved April 11, 2003 from http://www.web3d.org/WorkingGroups/vrml-eai/.

Rikk, C. (1997), Gavin, B., “The Annotated VRML 2.0 Reference Manual” Addison-Wesley Longman Ltd., Essex, UK.

Serway, R.A. (2000), R.J. Beichner, "Physics for Scientist and Engineers", 5th. Ed., Saunders College Publishing, Philadelphia.

Sokoloff D. R. (1997), and Thornton, R. K., "Using interactive lecture demonstrations to create an active learning environment" The Physics Teacher Vol.35, pp.340.

Vidimce, K. (2000), Foley, J. T., Banks, D. C., Chi, Y. T., and Mzoughi, "WebTOP: Interactive Optics on the Web," The Web3D/VRML 2000 Symposium, Monterey, California, 21-24 February 2000, pp. 125-129.

XML (2003), Extensible Markup Language Specifications. Retrieved April 11, 2003 from http://www.w3.org/XML/.

\section{ACKNOWLEDGMENT}

WebTOP is supported by the National Science Foundation Grant DUE 9950569 and DUE 0231217.

Taha Mzoughi

Department of Physics and Astronomy

Mississippi State University

Mississippi State, MS 39762, USA

Email : mzoughi@ra.msstate.edu 
John T. Foley

Department of Physics and Astronomy

Mississippi State University

Mississippi State, MS 39762, USA

Email : jtf1@ra.msstate.edu

Davis Herring

Department of Physics and Astronomy

Mississippi State University

Mississippi State, MS 39762, USA

Email :sdh6@msstate.edu

Matt Morris

Department of Computer Science

Mississippi State University

Mississippi State, MS 39762, USA

Email :mjm9@msstate.edu

Ben Wyser

Department of Computer Science

Mississippi State University

Mississippi State, MS 39762, USA

Email : bjw2@msstate.edu 